\title{
LINEAR RELATIONS AMONG HOLOMORPHIC QUADRATIC DIFFERENTIALS AND INDUCED SIEGEL'S METRIC ON $\mathcal{M}_{g}$
}

\author{
MARCO MATONE AND ROBERTO VOLPATO
}

\begin{abstract}
We derive the explicit form of the $(g-2)(g-3) / 2$ linearly independent relations among the products of pairs in a basis of holomorphic abelian differentials in the case of canonical curves of genus $g \geq 4$. It turns out that Petri's relations remarkably match in determinantal conditions. We explicitly express the volume form on the moduli space $\hat{\mathcal{M}}_{g}$ of canonical curves induced by the Siegel metric, in terms of the period Riemann matrix only. By the Kodaira-Spencer map, the relations lead to an expression of the induced Siegel metric on $\hat{\mathcal{M}}_{g}$, that corresponds to the square of the Bergman reproducing kernel. A key role is played by distinguished bases for holomorphic differentials whose properties also lead to an immediate derivation of Fay's trisecant identity.
\end{abstract}

\section{INTRODUCTION}

In spite of the remarkable progresses in understanding the Schottky problem, the characterization of the Schottky locus as the zero set of modular forms on the Siegel's upper half-space remains a fundamental open question. Such a question is strictly related to the problem of characterizing the Schottky locus by means of $(g-2)(g-3) / 2$ linearly independent conditions. As suggested also by Mumford (see pg. 241 of [1]), a possible solution of that problem should follow by a deeper understanding of Petri's construction 2, 3. Actually, since Petri's theorem determines the ideal of canonical curves of genus $g \geq 4$ by means of linear relations among holomorphic abelian differentials, it seems the natural framework for such an investigation.

Let $\left\{\eta_{1}, \ldots, \eta_{g}\right\}$ be the Petri's basis for $H^{0}\left(K_{C}\right)$, with $C$ a canonical curve of genus $g$. In Petri's work the coefficients $C_{i j}^{k}$ in the relationships among quadratic differentials $\sum_{i, j}^{g} C_{i j}^{k} \eta_{i} \eta_{j}=0, k=1, \ldots,(g-2)(g-3) / 2$, are not determined. Finding such coefficients is a necessary condition for an explicit characterization of the ideal of canonical curves. Here, we express Petri's relation in determinantal form, so that, besides the explicit determination of the coefficients, it is shown that the locus of canonical curves corresponds to a determinantal variety.

We introduce modular invariant bases for holomorphic differentials, leading to a refinement of Petri's basis and to an immediate derivation of Fay's trisecant identity 4. A key point is the introduction of a indexing, which includes the combinatorics of the Petri construction, mapping the components of matrices in the Siegel upper

Date: September 2006.

2000 Mathematics Subject Classification. Primary 30F30.

Work partially supported by the European Community's Human Potential Programme under contract MRTN-CT-2004-005104 "Constituents, Fundamental Forces and Symmetries of the Universe". 
half-space to vector components. This provides the volume form on the moduli space $\hat{\mathcal{M}}_{g}$ of canonical curves induced by the Siegel metric which, remarkably, is expressed in terms of the period Riemann matrix only. By the Kodaira-Spencer map, the above relations lead to an expression of the metric on $\hat{\mathcal{M}}_{g}$, induced by the Siegel metric, that corresponds to the square of the Bergman reproducing kernel.

In the case of branched covering of the torus, corresponding to Jacobians with a distinguished complex multiplication [5, the derived relations should lead to identities of number theoretical interest. Our results, of interest also in superstring theory [6], provide the key for the $(g-2)(g-3) / 2$ combinatorial $\theta$-identities in [7].

\section{Determinantal Characterization of CANONiCAL CURVES}

Let $C$ be a canonical curve of genus $g \geq 4$ and $\left\{\omega_{i}\right\}_{i \in I_{g}}, I_{n}:=\{1, \ldots, n\}$, a basis of $H^{0}\left(K_{C}\right)$, with $K_{C}$ the canonical line bundle of $C$. Denote by $\hat{\mathcal{M}}_{g}$ the corresponding locus in the moduli space $\mathcal{M}_{g}$ of compact Riemann surfaces. Each element of $H^{0}\left(K_{C}^{2}\right)$ can be written as a linear combination of the $M:=g(g+1) / 2$ elements in

$$
\mathcal{S}:=\left\{\omega_{i} \omega_{j} \mid i \leq j \in I_{g}\right\}
$$

Since $N:=h^{0}\left(K_{C}^{2}\right)=3 g-3$, there are $M-N=(g-2)(g-3) / 2$ linearly independent relations among the quadratic differentials $\omega_{i} \omega_{j}$.

Let $p_{1}, \ldots, p_{g}$ and $q_{1}, \ldots, q_{2 g-2}$ be two sets of arbitrary points on $C$. Choose a local trivialization of the canonical line bundle and set

$$
a_{i j, r}:=\operatorname{det} \omega\left(p_{1}, \ldots, p_{i-1}, q_{r}, p_{i+1}, \ldots, p_{g}\right) \operatorname{det} \omega\left(p_{1}, \ldots, p_{j-1}, q_{r}, p_{j+1}, \ldots, p_{g}\right),
$$

where $\operatorname{det} \omega\left(x_{1}, \ldots, x_{g}\right):=\operatorname{det} \omega_{i}\left(x_{j}\right)$. Set

$$
A(k, l):=\left(\begin{array}{ccccccc}
a_{12,1} & \ldots & a_{1 g, 1} & a_{23,1} & \ldots & a_{2 g, 1} & a_{k l, 1} \\
a_{12,2} & \ldots & a_{1 g, 2} & a_{23,2} & \ldots & a_{2 g, 2} & a_{k l, 2} \\
\vdots & \ddots & \vdots & \vdots & \ddots & \vdots & \vdots \\
a_{12,2 g-2} & \ldots & a_{1 g, 2 g-2} & a_{23,2 g-2} & \ldots & a_{2 g, 2 g-2} & a_{k l, 2 g-2}
\end{array}\right),
$$

$3 \leq k<l \leq g, g \geq 4$.

Theorem 2.1.

$$
\operatorname{det} A(k, l)=0, \quad 3 \leq k<l \leq g
$$

Set

$$
\Delta_{m n}:=(-)^{m+n} \operatorname{det}_{\substack{i \neq m \\ j \neq n}} A(k, l)_{i j}, \quad D_{p q}:=(-)^{p+q} \operatorname{det}_{\substack{i \neq p \\ j \neq q}} \omega_{j}\left(p_{i}\right),
$$

and denote by $A_{i j, r}(k, l), i, j \in I_{g}, r \in I_{2 g-2}$, the matrix obtained from $A(k, l)$ by replacing the row $\left(a_{12, r}, \ldots, a_{k l, r}\right)$ with $\left(D_{1 i} D_{2 j}, \ldots, D_{k i} D_{l j}\right)$.

Corollary 2.2. For each $r \in I_{2 g-2}$, the following relations

$$
\sum_{i, j=1}^{g} \frac{\operatorname{det} A_{i j, r}(k, l)}{\Delta_{r 2 g-2}} \omega_{i} \omega_{j}=0
$$

$3 \leq k<l \leq g$, provide $(g-2)(g-3) / 2$ linearly independent conditions on $\mathcal{S}$ which are independent of the points $q_{i}, i \in I_{2 g-2}$. 


\section{Distinguished Bases of $H^{0}\left(K_{C}^{n}\right)$}

Set $N_{n}:=(2 n-1)(g-1)+\delta_{1 n}, n \geq 1$, with $\delta_{i j}$ the Kronecker delta. Note that $N_{1}=g$ and $N_{2} \equiv N$. Fix a system of local coordinates on $C$.

Proposition 3.1. Fix $n \geq 1$ and let $p_{1}, \ldots, p_{N_{n}}$ be a set of points in $C$ such that

$$
\operatorname{det} \phi\left(p_{1}, \ldots, p_{N_{n}}\right) \neq 0,
$$

for an arbitrary basis $\left\{\phi_{i}\right\}_{i \in I_{N_{n}}}$ of $H^{0}\left(K_{C}^{n}\right)$. Then

$$
\gamma_{i}^{n}(z):=\frac{\operatorname{det} \phi\left(p_{1}, \ldots, p_{i-1}, z, p_{i+1}, \ldots, p_{N_{n}}\right)}{\operatorname{det} \phi\left(p_{1}, \ldots, p_{N_{n}}\right)},
$$

$i \in I_{N_{n}}$, determines a basis of $H^{0}\left(K_{C}^{n}\right)$ which is independent of the choice of the basis $\left\{\phi_{i}\right\}_{i \in I_{N_{n}}}$ and, up to normalization, of the local coordinates on $C$.

Proof. The matrix $[\phi]_{i j}:=\phi_{i}\left(p_{j}\right)$ is non-singular. Then $\gamma_{i}^{n}(z)=\sum_{j}[\phi]_{i j}^{-1} \phi_{j}(z)$, $i \in I_{N_{n}}$, is a basis of $H^{0}\left(K_{C}^{n}\right)$.

Note that $\gamma_{i}^{n}\left(p_{j}\right)=\delta_{i j}$, furthermore

$$
\operatorname{det} \gamma^{n}\left(p_{1}, \ldots, p_{j-1}, z, p_{j+1}, \ldots, p_{N_{n}}\right)=\gamma_{j}^{n}(z) .
$$

Remark 1. As we will see, the Fay trisecant identity [4] directly follows by expressing Eq. (3.1) in terms of theta functions.

For $n=1$, the choice of $g$ points $p_{1}, \ldots, p_{g} \in C$, with det $\omega_{i}\left(p_{j}\right) \neq 0$, determines the basis $\left\{\sigma_{i}\right\}_{i \in I_{g}}$ of $H^{0}\left(K_{C}\right)$, where

$$
\sigma_{i}(z):=\gamma_{i}^{1}(z), \quad i \in I_{g} .
$$

We now introduce a refinement of Petri's basis for $H^{0}\left(K_{C}^{2}\right)$ [2, 3] which provides a modular invariant construction. Let us assume that the points $p_{1}, \ldots, p_{g}$ are in "general position" and that the effective divisor $\left(\sigma_{1}\right)+\left(\sigma_{2}\right)-\sum_{i=3}^{g} p_{i}$ consists of $3 g-3$ distinct points. Consider the following $M$ elements of $H^{0}\left(K_{C}^{2}\right)$

$$
v_{i}:= \begin{cases}\sigma_{i}^{2}, & i \in I_{g}, \\ \sigma_{j+k} \sigma_{j}, & i=k+j(2 g-j+1) / 2, \quad j \in I_{g-1}, \quad k \in I_{g-j} .\end{cases}
$$

Proposition 3.2. $\left\{v_{i}\right\}_{i \in I_{N}}$ is a basis of $H^{0}\left(K_{C}^{2}\right)$.

Proof. Set $D:=\sum_{i=3}^{g} p_{i}$ and let us consider the effective divisors $D_{i}:=\left(\sigma_{i}\right)-D$, $i=1,2$. Let us first prove that $\sigma_{i}$ is the unique 1-differential, up to normalization, vanishing at $D_{i}, i=1,2$. Any 1-differential $\sigma_{i}^{\prime} \in H^{0}\left(K_{C}\right)$ vanishing at $D_{i}$, corresponds to an element $\sigma_{i}^{\prime} / \sigma_{i}$ of $H^{0}(\mathcal{O}(D))$, the space of meromorphic functions $f$ on $C$ such that $(f)+D$ is an effective divisor. Suppose that there exists $\sigma_{i}^{\prime}$ such that $\sigma_{i}^{\prime} / \sigma_{i}$ is not a constant, so that $h^{0}(\mathcal{O}(D)) \geq 2$. By Riemann-Roch theorem

$$
h^{0}\left(K_{C} \otimes \mathcal{O}(-D)\right)=h^{0}(\mathcal{O}(D))-\operatorname{deg} D-1+g \geq 3
$$

so that there exist at least 3 linearly independent 1-differentials vanishing at $D$ and, in particular, there exists a linear combination of such differentials vanishing at $p_{1}, \ldots, p_{g}$. This implies that $\operatorname{det} \eta\left(p_{1}, \ldots, p_{g}\right)=0$ for an arbitrary basis $\left\{\eta_{i}\right\}_{i \in I_{g}}$ of $H^{0}\left(K_{C}\right)$, contradicting the hypotheses. Fix $\zeta_{i}, \zeta_{1 i}, \zeta_{2 i} \in \mathbb{C}$ such that

$$
\sum_{i=3}^{g} \zeta_{i} \sigma_{i}^{2}+\sum_{i=1}^{g} \zeta_{2 i} \sigma_{1} \sigma_{i}+\sum_{i=2}^{g} \zeta_{1 i} \sigma_{2} \sigma_{i}=0
$$


Evaluating this relation at the point $p_{j}, 3 \leq j \leq g$, yields $\zeta_{j}=0$. Set

$$
t_{1}:=-\sum_{j=2}^{g} \zeta_{1 j} \sigma_{j}, \quad t_{2}:=\sum_{j=1}^{g} \zeta_{2 j} \sigma_{j},
$$

so that $\sigma_{1} t_{2}=\sigma_{2} t_{1}$. Since $D, D_{1}$ and $D_{2}$ consist of pairwise distinct points, $t_{i}$ vanishes at $D_{i}, i=1,2$ and then $t_{1} / \sigma_{1}=t_{2} / \sigma_{2}=\zeta \in \mathbb{C}$. By (3.4)

$$
\zeta \sigma_{1}+\sum_{j=2}^{g} \zeta_{1 j} \sigma_{j}=0, \quad \zeta \sigma_{2}-\sum_{k=1}^{g} \zeta_{2 k} \sigma_{k}=0,
$$

and, by linear independence of $\sigma_{1}, \ldots, \sigma_{g}$, we have $\zeta=\zeta_{1 j}=\zeta_{2 k}=0,2 \leq j \leq g$, $k \in I_{g}$.

\section{Proofs of Theorem 2.1 and Corollary 2.2}

Let $W(P)$ be the Wronskian $W\left(v_{1}, \ldots, v_{N}\right)(P)$ of the basis $\left\{v_{i}\right\}_{i \in I_{N}}$ at a generic point $P \in C$, and $\hat{W}_{i j}(P):=W\left(v_{1}, \ldots, v_{i-1}, v_{j}, v_{i+1}, \ldots, v_{N}\right)(P)$.

Lemma 4.1. The $(g-2)(g-3) / 2$ linearly independent relations

$$
v_{i}(z) W(P)=\sum_{j=1}^{N} v_{j}(z) \hat{W}_{j i}(P),
$$

$i=N+1, \ldots, M$, hold $\forall z \in C$.

Proof. Immediate consequence of the Cramer rule.

Remark 2. The ratio $\hat{W}_{i j}(P) / W(P)$ does not depend on $P$.

Remark 3. Since for $i \in I_{g}$

$$
\begin{cases}v_{j}\left(p_{i}\right)=\delta_{j i}, & j \in I_{g}, \\ v_{j}\left(p_{i}\right)=0, & j=g+1, \ldots, M,\end{cases}
$$

it follows that for $z=p_{i}$ Eq.(4.1) gives $\hat{W}_{i j}(P)=0$ for $i \in I_{g}$ and $j=N+1, \ldots, M$.

Remark 4. The Wronskians in the expansion (4.1) can be replaced by the corresponding determinant $\operatorname{det} v_{j}\left(x_{i}\right)$, where $x_{1}, \ldots, x_{3 g-3}$ are arbitrary points on $C$.

Proof of Theorem 2.1. Assume that $\operatorname{det} \omega\left(p_{1}, \ldots, p_{g}\right) \neq 0$. Define

$$
x_{i}:= \begin{cases}p_{i}, & i \in I_{g}, \\ q_{i-g}, & i=g+1, \ldots, N+1 .\end{cases}
$$

Fix $k, l$ with $3 \leq k<l \leq g$ and consider the matrix

$$
\left(\begin{array}{cccc}
v_{1}\left(x_{1}\right) & \ldots & v_{N}\left(x_{1}\right) & \sigma_{k}\left(x_{1}\right) \sigma_{l}\left(x_{1}\right) \\
\vdots & \ddots & \vdots & \vdots \\
v_{1}\left(x_{N+1}\right) & \ldots & v_{N}\left(x_{N+1}\right) & \sigma_{k}\left(x_{N+1}\right) \sigma_{l}\left(x_{N+1}\right)
\end{array}\right) .
$$

By (4.2), this matrix has $\operatorname{diag}(1, \ldots, 1)$ in the $g \times g$ upper left corner, 0 in the $g \times(2 g-2)$ upper right corner and $\left(\operatorname{det} \omega\left(p_{1}, \ldots, p_{g}\right)\right)^{-2} A(k, l)$ in the $(2 g-2) \times$ $(2 g-2)$ lower right corner. On the other hand, by Lemma 4.1 and by Remark 4 
the determinant of this matrix vanishes. Since such relations hold for $\left(p_{1}, \ldots, p_{g}\right)$ in a dense subset of $C^{g}$, they hold $\forall\left(p_{1}, \ldots, p_{g}\right) \in C^{g}$ and the theorem follows.

Proof of Corollary 2.2. Divide the relations in Theorem 2.1 by $\Delta_{i 2 g-2}$ and note that

$$
a_{m n, r}=\sum_{i, j=1}^{g} D_{m i} D_{n j} \omega_{i}\left(q_{r}\right) \omega_{j}\left(q_{r}\right) .
$$

Independence of the points $q_{i}, i \in I_{2 g-2}$, follows by noting that the coefficients of $\omega_{i} \omega_{j}$ in the relations are functions of $q_{i}$ with no zeroes or poles.

Define

$$
\left(\mathbf{1}_{i}, 2_{i}\right):=\left\{\begin{array}{cc}
(i, i), & 1 \leq i \leq g \\
(1, i-g+1), & g+1 \leq i \leq 2 g-1 \\
(2, i-2 g+3), & 2 g \leq i \leq 3 g-3 \\
\vdots & \vdots \\
(g-1, g), & i=g(g+1) / 2
\end{array}\right.
$$

so that $\mathbf{1}_{i} 2_{i}$ is the $i$-th element in the $M$-tuple $(11,22, \ldots, g g, 12, \ldots, 1 g, 23, \ldots)$. $\forall u \in \mathbb{C}^{g}$ and for all the $g \times g$ matrices $A$, set

$$
u u_{i}:=u_{1_{i}} u_{2_{i}}, \quad(A A)_{i j}:=\frac{A_{1_{i 1_{j}}} A_{2_{i} 2_{j}}+A_{1_{i 2_{j}}} A_{2_{i 1_{j}}}}{1+\delta_{1_{j} 2_{j}}}, \quad A_{i}:=A_{1_{i 2_{i}}},
$$

$i, j \in I_{M}$. In the following we will repeatedly make use of the identities

$$
\sum_{i, j=1}^{g} f(i, j)=\sum_{i \leq j}^{g} \frac{f(i, j)+f(j, i)}{1+\delta_{i j}}=\sum_{k=1}^{M} \frac{f\left(\mathbf{1}_{k}, 2_{k}\right)+f\left(2_{k}, \mathbf{1}_{k}\right)}{1+\delta_{\mathbf{1}_{k} 2_{k}}} .
$$

In particular, if $f(i, j)=f(j, i)$, then

$$
\sum_{i, j=1}^{g} f(i, j)=\sum_{k=1}^{M}\left(2-\delta_{\mathbf{1}_{k} 2_{k}}\right) f\left(\mathbf{1}_{k}, 2_{k}\right)
$$

where we used the identity

$$
2-\delta_{i j}=\frac{2}{1+\delta_{i j}} .
$$

With this notation, and observing that $\sigma_{i}=\sum_{j=1}^{g}[\omega]_{i j}^{-1} \omega_{j}$, we have

$$
v_{i}=\sigma \sigma_{i}=\sum_{j=1}^{M}\left([\omega]^{-1}[\omega]^{-1}\right)_{i j} \omega \omega_{j}, \quad i \in I_{M}
$$

Set $w_{i j}:=W_{i j} / W$, where $W_{i j}(P):=W\left(v_{1}, \ldots, v_{i-1}, \omega \omega_{j}, v_{i+1}, \ldots, v_{N}\right)(P)$, and note that

$$
\omega \omega_{i}=\sum_{j=1}^{N} w_{j i} v_{j}, \quad i \in I_{M}
$$




\section{Siegel's induced measure on $\hat{\mathcal{M}}_{g}$ And Bergman reproducing Kernel}

Let

$$
\mathfrak{H}_{g}:=\left\{\left.Z \in M_{g}(\mathbb{C})\right|^{t} Z=Z, Y>0\right\}, \quad Y:=\Im Z,
$$

be the Siegel upper half-space and $\left\{\alpha_{1}, \ldots, \alpha_{g}, \beta_{1}, \ldots, \beta_{g}\right\}$ a symplectic basis of $H_{1}(C, \mathbb{Z})$. Denote by $\left\{\omega_{i}\right\}_{i \in I_{g}}$ the basis of $H^{0}\left(K_{C}\right)$, dual of $H_{1}(C, \mathbb{Z})$, so that $\oint_{\alpha_{i}} \omega_{j}=\delta_{i j}, i, j \in I_{g}$. Let $\tau_{i j}:=\oint_{\beta_{i}} \omega_{j} \in \mathfrak{H}_{g}$ be the Riemann period matrix of $C$. Under the symplectic transformation

$$
\left(\begin{array}{c}
\tilde{\alpha} \\
\tilde{\beta}
\end{array}\right)=\left(\begin{array}{ll}
D & C \\
B & A
\end{array}\right)\left(\begin{array}{l}
\alpha \\
\beta
\end{array}\right), \quad\left(\begin{array}{ll}
A & B \\
C & D
\end{array}\right) \in S p(2 g, \mathbb{Z}),
$$

we have $\tilde{\omega}={ }^{t}(C \tau+D) \cdot \omega$, with $\tilde{\tau}_{i j}$ and $\tau_{i j}$ related by the modular transformation

$$
\tilde{\tau}=(A \tau+B) \cdot(C \tau+D)^{-1} .
$$

Note that the basis $\left\{\sigma_{i}\right\}_{i \in I_{g}}$, defined in Eq.(3.3), is independent of the choice of the basis of $H_{0}\left(K_{C}\right)$ and therefore is modular invariant.

The Siegel metric

$$
d s^{2}:=\operatorname{Tr}\left(Y^{-1} d Z Y^{-1} d \bar{Z}\right)
$$

defines the volume form

$$
d \nu=\frac{i^{M}}{2^{g}} \frac{\bigwedge_{i \leq j}^{g}\left(\mathrm{~d} Z_{i j} \wedge \mathrm{d} \bar{Z}_{i j}\right)}{\operatorname{det} Y^{g+1}} .
$$

We use the indexing introduced in Eq.(4.3) to express the Siegel metric on $\mathfrak{H}_{g}$ where now the matrix elements $Z_{i j}, i, j \in I_{M}$, are seen as the components of the $M$-dimensional vectors $Z:=\left(Z_{1}, \ldots, Z_{M}\right)$.

\section{Proposition 5.1.}

$$
d s^{2}=\sum_{i, j=1}^{M} g_{i j} d Z_{i} d \bar{Z}_{j}
$$

where

$$
g_{i j}:=\left(2-\delta_{1_{i 2}}\right)\left(Y^{-1} Y^{-1}\right)_{i j} .
$$

Proof. By (4.4) and (4.5)

$$
\begin{aligned}
d s^{2} & =\sum_{i, j, k, l=1}^{g} Y_{i j}^{-1} d Z_{j k} Y_{k l}^{-1} d \bar{Z}_{l i} \\
& =\sum_{i, l=1}^{g} d \bar{Z}_{l i} \sum_{m=1}^{M} \frac{Y_{i_{m}}^{-1} Y_{l_{m}}^{-1}+Y_{i_{2}}^{-1} Y_{l_{\mathbf{1}_{m}}}^{-1}}{1+\delta_{\mathbf{1}_{m} 2_{m}}} d Z_{\mathbf{1}_{m} 2_{m}} \\
& =\sum_{m, n=1}^{M}\left(2-\delta_{\mathbf{1}_{n} 2_{n}}\right) d \bar{Z}_{\mathbf{1}_{n} 2_{n}} \frac{Y_{\mathbf{1}_{n} \mathbf{1}_{m}}^{-1} Y_{\mathbf{2}_{n} \mathbf{2}_{m}}^{-1}+Y_{\mathbf{1}_{n} 2_{m}}^{-1} Y_{\mathbf{2}_{n} \mathbf{1}_{m}}^{-1}}{1+\delta_{\mathbf{1}_{m} 2_{m}}} d Z_{\mathbf{1}_{m} 2_{m}} \\
& =\sum_{m, n=1}^{M}\left(2-\delta_{\mathbf{1}_{n} 2_{n}}\right)\left(Y^{-1} Y^{-1}\right)_{n m} d Z_{m} d \bar{Z}_{n} .
\end{aligned}
$$


Let $k$ be the Kodaira-Spencer map identifying the quadratic differentials on $C$ with the fiber of the cotangent of the Teichmüller space at $C$. We have

$$
k\left(\omega_{i} \omega_{j}\right)=(2 \pi i)^{-1} d \tau_{i j} .
$$

By Corollary 2.2 it follows that

$$
\sum_{i, j=1}^{g} \frac{\operatorname{det} A_{i j, r}(k, l)}{\Delta_{r 2 g-2}} d \tau_{i j}=0
$$

$3 \leq k<l \leq g$. Set $d \tau_{i}:=d \tau_{1_{i} 2_{i}}, i \in I_{M}$. Eq.(5.3) yields an explicit expression for the volume form on $\hat{\mathcal{M}}_{g} \hookrightarrow \mathfrak{H}_{g} / S p(2 g, \mathbb{Z})$ induced by the modular invariant Siegel metric on $\mathfrak{H}_{g}$. Set $\tau_{2}:=\Im \tau$, and let $\left|\tau_{2}^{-1} \tau_{2}^{-1}\right|_{j_{1} \ldots j_{N}}^{i_{1} \ldots i_{N}}$, with $i_{k}, j_{k}, k \in I_{N}$, distinct elements of $I_{M}$, be the determinant of the $N \times N$ submatrix of $\left(\tau_{2}^{-1} \tau_{2}^{-1}\right)_{i j}$, built by taking the rows $i_{1}, \ldots, i_{N}$ and the columns $j_{1}, \ldots, j_{N}$.

Theorem 5.2. The volume form on $\hat{\mathcal{M}}_{g}$ induced by the Siegel metric is

$$
d \nu_{\mid \hat{\mathcal{M}}_{g}}=\left(\frac{i}{2}\right)^{N} \sum_{\substack{i_{N}>\ldots>i_{1}=1 \\ j_{N}>\ldots>j_{1}=1}}^{M}\left|\tau_{2}^{-1} \tau_{2}^{-1}\right|_{j_{1} \ldots j_{N}}^{i_{1} \ldots i_{N}} \prod_{k=1}^{N}\left(2-\delta_{1_{i_{k} 2_{i_{k}}}}\right) \bigwedge_{1}^{N}\left(d \tau_{i_{k}} \wedge d \bar{\tau}_{j_{k}}\right)
$$

so that

$$
\operatorname{Vol}\left(\hat{\mathcal{M}}_{g}\right)=\int_{\hat{\mathcal{M}}_{g}} d \nu_{\mid \hat{\mathcal{M}}_{g}}
$$

Proof. Let

$$
\omega:=\frac{i}{2} \sum_{i, j=1}^{M} g_{i j} d Z_{i} \wedge d \bar{Z}_{j},
$$

be the $(1,1)$-form associated to the Siegel metric on $\mathfrak{H}_{g}$. By Wirtinger's theorem [8, the volume form on a $d$-dimensional complex submanifold $S$ is

$$
\frac{1}{d !} \omega^{d},
$$

so that the volume of $S$ is expressed as the integral over $S$ of a globally defined differential form on $\mathfrak{H}_{g}$. Set $g_{i j}^{\tau}:=\left(2-\delta_{1_{i} 2_{i}}\right)\left(\tau_{2}^{-1} \tau_{2}^{-1}\right)_{i j}, i, j \in I_{M}$, and note that

$$
\begin{aligned}
d \nu_{\mid \hat{\mathcal{M}}_{g}} & =\frac{i^{N}}{2^{N} N !} \sum_{\substack{i_{1}, \ldots, i_{N}=1 \\
j_{1}, \ldots, j_{N}=1}}^{M} \prod_{k=1}^{N} g_{i_{k} j_{k}}^{\tau} \bigwedge_{k=1}^{N}\left(d \tau_{i_{k}} \wedge d \bar{\tau}_{j_{k}}\right) \\
& =\frac{i^{N}}{2^{N} N !} \sum_{\substack{i_{N}<\ldots<i_{1}=1 \\
j_{N}<\ldots<j_{1}=1}}^{M} \sum_{r, s \in \mathcal{P}_{N}} \epsilon(r) \epsilon(s) \prod_{k=1}^{N} g_{i_{r(k)} j_{s(k)}} \bigwedge_{k=1}^{N}\left(d \tau_{i_{k}} \wedge d \bar{\tau}_{j_{k}}\right),
\end{aligned}
$$

where $\mathcal{P}_{N}$ is the group of permutations of $N$ elements and $\epsilon(s)$ is the sign of the permutation $s$. The theorem then follows by the identity

$$
\sum_{r, s \in \mathcal{P}_{N}} \epsilon(r) \epsilon(s) \prod_{k=1}^{N} g_{i_{r(k)} j_{s(k)}}^{\tau}=N !\left|\tau_{2}^{-1} \tau_{2}^{-1}\right|_{j_{1} \ldots j_{N}}^{i_{1} \ldots i_{N}} \prod_{k=1}^{N}\left(2-\delta_{1_{i_{k} 2_{k}}}\right) .
$$


Petri's basis of $H^{2}\left(K_{C}^{2}\right)$ corresponds, through the Kodaira-Spencer map, to a basis for the cotangent space of the Teichmüller space. Setting $d \Xi_{i}:=2 \pi i k\left(v_{i}\right)$, $i \in I_{N}$, it follows by Eq.(4.7) that

$$
d \tau_{i}=\sum_{j=1}^{N} w_{j i} d \Xi_{j}, \quad i \in I_{M} .
$$

Corollary 5.3. Fix the points $p_{1}, \ldots, p_{g} \in C$ in general position, so that $\left\{v_{i}\right\}_{i \in I_{N}}$, is a basis of $H^{0}\left(K_{C}^{2}\right)$. The metric on $\hat{\mathcal{M}}_{g}$ induced by the Siegel metric is

$$
d s_{\mid \hat{\mathcal{M}}_{g}}^{2}=\sum_{i, j=1}^{N} g_{i j}^{\Xi} d \Xi_{i} d \bar{\Xi}_{j}
$$

where $g_{i j}^{\Xi}:=\sum_{k, l=1}^{M}\left(2-\delta_{1_{k} 2_{k}}\right) w_{i k}\left(\tau_{2}^{-1} \tau_{2}^{-1}\right)_{k l} \bar{w}_{j l}$.

Proof. Immediate.

By using a suitable basis of $H^{0}\left(K_{C}^{2}\right)$ and its image under the Kodaira-Spencer map, it turns out that the metric $g$ is related to the Bergman reproducing kernel. Fix the points $z_{1}, \ldots, z_{N} \in C$ satisfying the conditions of Proposition 3.1. The basis $\left\{\gamma_{i}\right\}_{i \in I_{N}}$ of $H^{0}\left(K_{C}^{2}\right)$, with $\gamma_{i} \equiv \gamma_{i}^{2}, i \in I_{N}$, defined by Eq.(3.1) in the case $n=2$, satisfies the relations

$$
\omega \omega_{i}=\sum_{j=1}^{N} \omega \omega_{i}\left(z_{j}\right) \gamma_{j}, \quad v_{i}=\sum_{j=1}^{N} v_{i}\left(z_{j}\right) \gamma_{j}, \quad i \in I_{M} .
$$

Set $\Gamma_{i}:=(2 \pi i)^{-1} k\left(\gamma_{i}\right)$ and $[v]_{i j}:=v_{i}\left(z_{j}\right), i, j \in I_{N}$.

Theorem 5.4.

$$
d s_{\mid \hat{\mathcal{M}}_{g}}^{2}=\sum_{i, j=1}^{N} B^{2}\left(z_{i}, \bar{z}_{j}\right) d \Gamma_{i} d \bar{\Gamma}_{j},
$$

in particular, the Siegel induced modular invariant volume form on $\hat{\mathcal{M}}_{g}$ is

$$
d \nu_{\mid \hat{\mathcal{M}}_{g}}=\left(\frac{i}{2}\right)^{N} \operatorname{det} B^{2}\left(z_{i}, \bar{z}_{j}\right) \bigwedge_{1}^{N}\left(d \Gamma_{i} \wedge d \bar{\Gamma}_{i}\right)
$$

where

$$
B(z, \bar{w}):=\sum_{i, j=1}^{g} \omega_{i}(z)\left(\tau_{2}^{-1}\right)_{i j} \bar{\omega}_{j}(w),
$$

$z, w \in C$, is the Bergman reproducing kernel.

Proof. Use $d \tau_{i}=\sum_{j=1}^{N} \omega \omega_{i}\left(z_{j}\right) d \Gamma_{j}, i \in I_{g}$, and the identity

$$
\sum_{k, l=1}^{M}\left(2-\delta_{1_{k} 2_{k}}\right) \omega \omega_{k}\left(z_{i}\right)\left(\tau_{2}^{-1} \tau_{2}^{-1}\right)_{k l} \bar{\omega} \bar{\omega}_{l}\left(z_{j}\right)=B^{2}\left(z_{i}, \bar{z}_{j}\right), \quad i, j \in I_{N}
$$

Note that by (5.8) $\sum_{k, l=1}^{N}[v]_{k i} g_{k l}^{\Xi}[\bar{v}]_{l j}=B^{2}\left(z_{i}, \bar{z}_{j}\right)$, which also follows by (4.7). 


\section{FAY'S TRISECANT IDENTITY FROM the Distinguished Basis of $H^{0}\left(K_{C}^{n}\right)$}

Let $I_{i}(p):=\int_{p_{0}}^{p} \omega_{i}, p_{0}, p \in C, i \in I_{g}$, be the Abel-Jacobi map, which extends to a map from divisors of $C$ to the Jacobian $J(C):=\mathbb{C}^{g} /\left(\mathbb{Z}_{g}+\tau \mathbb{Z}^{g}\right)$. We consider Riemann $\theta$-functions $\theta(D+e):=\theta(I(D)+e, \tau), e \in J(C)$, evaluated at some 0-degree divisor $D$ of $C$. By the Riemann vanishing theorem, there is a divisor class $\Delta$ of degree $g-1$ with $2 \Delta=K$, such that $-I(\Delta)$ is the vector of Riemann constants. Let $E(z, w)$ be the prime form, and set

$$
\sigma(z):=\exp \left(-\sum_{i=1}^{g} \oint_{\alpha_{i}} \omega_{i}(w) \ln E(z, w)\right) .
$$

Proposition 6.1. Fix $n \in \mathbb{N}$ and let $\left\{\phi_{i}^{n}\right\}_{i \in I_{N_{n}}}$ be an arbitrary basis of $H^{0}\left(K_{C}^{n}\right)$, $n \geq 1$. Let $y, x_{1}, \ldots, x_{N_{n}}$ be arbitrary points of $C$. Then, for $n=1$

$$
\operatorname{det} \phi_{i}^{1}\left(x_{j}\right)=\kappa_{1}\left[\phi^{1}\right] \frac{\theta\left(\sum_{1}^{g} x_{i}-y-\Delta\right) \prod_{i<j}^{g} E\left(x_{i}, x_{j}\right) \prod_{1}^{g} \sigma\left(x_{k}\right)}{\sigma(y) \prod_{1}^{g} E\left(y, x_{i}\right)},
$$

whereas for $n>1$

$$
\operatorname{det} \phi_{i}^{n}\left(x_{j}\right)=\kappa_{n}\left[\phi^{n}\right] \theta\left(\sum_{1}^{N_{n}} x_{i}-(2 n-1) \Delta\right) \prod_{i<j}^{N_{n}} E\left(x_{i}, x_{j}\right) \prod_{i=1}^{N_{n}} \sigma\left(x_{i}\right)^{2 n-1},
$$

where $\kappa_{1}\left[\phi^{1}\right]$ and $\kappa_{n}\left[\phi^{n}\right]$ are constants depending only on the choice of the bases.

Proof. $\kappa_{1}\left[\phi^{1}\right]$ is a nowhere vanishing section in $x_{j}, j \in I_{g}$, and $\theta\left(\sum_{1}^{g} x_{i}-y-\Delta\right)=0$ for $y=x_{1}, \ldots, x_{g}$, so that it is also a nowhere vanishing section in $y$ and since it has trivial monodromy it must be a constant. Eq.(6.2) follows by a similar proof.

Set $w:=\sum_{1}^{N_{n}} p_{i}-(2 n-1) \Delta, n>1$, and assume that $p_{1}, \ldots, p_{N_{n}} \in C$ satisfy the hypothesis of Proposition 3.1. By Proposition 6.1 we have

$$
\gamma_{i}^{n}(z)=\frac{\theta\left(w+z-p_{i}\right) \sigma(z)^{2 n-1} \prod_{\substack{k=1 \\ k \neq i}}^{N_{n}} E\left(z, p_{k}\right)}{\theta(w) \sigma\left(p_{i}\right)^{2 n-1} \prod_{\substack{k=1 \\ k \neq 1}}^{N_{n}} E\left(p_{i}, p_{k}\right)}, \quad i \in I_{N_{n}} .
$$

Theorem 6.2. Propositions 3.1 and 6.1 imply the Fay trisecant identity [4]

$$
\frac{\theta\left(w+\sum_{i=1}^{m}\left(x_{i}-y_{i}\right)\right) \prod_{i<j} E\left(x_{i}, x_{j}\right) E\left(y_{i}, y_{j}\right)}{\theta(w) \prod_{i, j} E\left(x_{i}, y_{j}\right)}=(-)^{\frac{m(m-1)}{2}} \operatorname{det}_{i j} \frac{\theta\left(w+x_{i}-y_{j}\right)}{\theta(w) E\left(x_{i}, y_{j}\right)},
$$

$m \geq 2, \forall x_{1}, \ldots, x_{m}, y_{1}, \ldots, y_{m} \in C, w \in J(C)$.

Proof. Fix $m \geq 2, x_{1}, \ldots, x_{m}, y_{1}, \ldots, y_{m} \in C$ and $w \in J(C)$, with $\theta(w) \neq 0$. Choose $y_{1}, \ldots, y_{m}$ distinct, otherwise the identity is trivial. Set $p_{i}:=y_{i}, i \in I_{m}$, and fix $n \in \mathbb{Z}, N_{n} \geq m$, and $p_{m+1}, \ldots, p_{N_{n}} \in C$, so that $w=\sum_{1}^{N_{n}} p_{i}-(2 n-1) \Delta$. By Jacobi inversion theorem such a choice is always possible, provided that $N_{n}-m \geq g$. It is also clear that, for $n$ large enough, $p_{m+1}, \ldots, p_{N_{n}}$ can be chosen pairwise distinct and distinct from $y_{1}, \ldots, y_{m}$. Eq.(6.2) implies that, by construction, $\operatorname{det} \phi_{i}^{n}\left(p_{j}\right) \neq 0$, for any basis $\left\{\phi_{i}^{n}\right\}_{i \in I_{N_{n}}}$ of $H^{0}\left(K_{C}^{n}\right)$, since the points $p_{1}, \ldots, p_{N_{n}}$ are pairwise distinct and $\theta(w) \neq 0$. Therefore, one can define a basis $\left\{\gamma_{i}^{n}\right\}_{i \in I_{N_{n}}}$ of $H^{0}\left(K_{C}^{n}\right)$ by (3.1) and consider $\operatorname{det} \gamma^{n}\left(x_{1}, \ldots, x_{m}, p_{m+1}, \ldots, p_{N_{n}}\right)$, which can be expressed by (6.3) or by (6.2), with $\kappa_{n}\left[\gamma^{n}\right]$ determined by applying (6.2) to $\operatorname{det} \gamma_{i}^{n}\left(p_{j}\right)=1$. Comparing these formulas the theorem follows. 


\section{REFERENCES}

1. D. Mumford, The red book of varieties and schemes, Springer Lecture Notes 1358 (1999).

2. K. Petri, Über die invariante darstellung algebraischer funktionen einer veränderlichen, Math. Ann. 88 (1922) 242 - 289.

3. E. Arbarello, M. Cornalba, P.A. Griffiths and J. Harris, Geometry of algebraic curves, I. Grundlehren Math. Wiss., vol. 267, Springer-Verlag, 1985.

4. J. Fay, Theta Functions on Riemann surfaces, Springer Lecture Notes 352.

5. M. Matone, Eigenfunctions of the Laplacian acting on degree zero bundles over special Riemann surfaces, Trans. Am. Math. Soc. 356 (2004) 2989 - 3004.

6. M. Matone and R. Volpato, Higher genus superstring amplitudes from the geometry of moduli spaces, Nucl. Phys. B 732 (2006) 321 - 340.

7. M. Matone and R. Volpato, Determinantal characterization of canonical curves and combinatorial theta identities, math.ag/0605734

8. P.A. Griffiths and J. Harris, Principles of Algebraic Geometry, Wiley, 1978.

Dipartimento di Fisica "G. Galilei" and Istituto Nazionale di Fisica Nucleare, Università di Padova, Via Marzolo, 8 -, 35131 Padova, Italy

E-mail address: matone@pd.infn.it

E-mail address: volpato@pd.infn.it 